\title{
Tangence
}

\section{Originaux et détraqués de la fin du XVIIIe siècle québécois}

\section{Bernard Andrès}

Numéro 57, mai 1998

Littérateurs atypiques et penseurs irréguliers

URI : https://id.erudit.org/iderudit/025968ar

DOI : https://doi.org/10.7202/025968ar

Aller au sommaire du numéro

Éditeur(s)

Tangence

ISSN

0226-9554 (imprimé)

1710-0305 (numérique)

Découvrir la revue

Citer cet article

Andrès, B. (1998). Originaux et détraqués de la fin du XVIIIe siècle québécois. Tangence, (57), 53-71. https://doi.org/10.7202/025968ar d'utilisation que vous pouvez consulter en ligne.

https://apropos.erudit.org/fr/usagers/politique-dutilisation/ 


\section{Originaux et détraqués de la fin du XVIII ${ }^{e}$ siècle québécois}

\section{Bernard Andrès, Université du Québec à Montréal — IREP}

C'est dans les marges de la marge littéraire francophone que gisent, à la fin du XvıIr ${ }^{\mathrm{e}}$ siècle, un certain nombre d'individus dont les noms sont à peine recensés et dont les œuvres, aux yeux des rares spécialistes égarés dans ces corpus, ne méritent pas même le nom d'œuvres. Des documents, tout au plus, des "curiosités" à peine littéraires que les historiens de l'imprimé mentionnent en passant, sourire en coin, et dont la critique, quand elle y condescend, dit bien du mal, faute de se donner le mal de les bien lire. Je parle du Québec, en marge de l'institution littéraire française. Je parle dans cette périphérie septentrionale d'une production marginale et d'une époque révolue : paléontologique, diront d'aucuns. Car ceux qui, à Paris comme à Montréal, croient à une littérature québécoise, ne situent son émergence qu'au lendemain de la "Révolution tranquille", dans la "modernité" des années 1960. Là enfin, selon des perspectives esthétique, sociologique, idéologique ou institutionnelle, nous aurions une masse critique d'œeuvres et de littérateurs patentés, un véritable appareil d'édition, une école, une université, des revues et tout le bataclan institutionnel.

Avant quoi, pas de salut, sauf à considérer tels auteurs, tels mouvements, telles chapelles comme autant d'étapes vers la consécration des lettres contemporaines. Avant quoi, oui, bien sûr, Nelligan. Oui, les Exotiques, oui, l'École littéraire de Montréal, au tournant du $\mathrm{xx}^{\mathrm{e}}$ siècle. Oui, peu avant, Philippe Aubert de Gaspé père, oui, l'abbé Casgrain et l'École patriotique de Québec. Oui, François-Xavier Garneau (mais le poète ou l'historien?). Oui, Joseph Doutre, à la rigueur. Oui mais, de grâce, finissons là. Aux yeux des modernistes, seules les lettres contemporaines sont dignes de passer à la postérité. Le $\mathrm{XIX}^{\mathrm{e}}$ siècle fait antichambre, de Philippe Aubert de Gaspé fils à Louis Fréchette, "notre Victor Hugo national" (hélas). L'auteur des contes et portraits intitulés Originaux et détraqués (1892) figure aussi parmi les gloires nationales de ce vestibule des lettres québécoises, limite ultime de 
54

toute légitimité. C'est pourtant à un autre avant-lieu littéraire que j'aimerais remonter pour présenter d'autres originaux plus ou moins détraqués (aux yeux des bien-pensants).

Mais, me demandé-je, pourquoi faire? À quoi bon? Aux seules fins de répondre à une commande ou à une demande? Pour sacrifier à un colloque de nébuleux noéticiens, dans une quête un peu gratuite d'alloxodoxies madonniennes? Et pourquoi pas - ceci dit sans cynisme aucun? Mais attention, s'intéresser aux marges de l'institution, interroger les périphéries, sonder l'atypie, traquer l'illégitime, c'est aussi conforter la légitimité de l'institution qui (truisme) se nourrit de ses propres exclusions, ses propres trublions, ses avant-gardes sans lesquels elle serait vouée - l'institution - à la sclérose (dixit Dubois, Bourdieu, Milot et consorts). Alors, si l'on ne tient pas à conforter le Même, comment aborder l'Autre en tenant le pari d'une irréductible illégitimité, bandée, tendue vers des pratiques limites, pathétiquement irrécupérables? Peut-être en choisissant une époque et une aire culturelles miraculeusement veuves d'institution: le Québec des années 1760 à 1800 . C'est ce que je propose ici en esquissant le tableau d'une époque et le portrait de quelques individus mutants et déviants à souhait.

C'est en travaillant à la biographie intellectuelle d'un certain Pierre de Sales Laterrière que j'ai découvert la galerie de portraits dont je vous parle aujourd'hui. Un mot pour commencer, sur Pierre de Sales Laterrière, "médecin et libre penseur " ${ }^{1}$. Un charlatan, diront les uns, un fieffé gascon pour les autres, un imposteur aux yeux de la plupart qui le soupçonnent de s'être forgé une généalogie, de s'être refait une identité au Québec. Plus j'avance dans le récit de sa vie, plus je suis fasciné par son inventivité, son originalité, mais aussi - fait paradoxal — sa conformité à l'esprit du temps. Atypique? Non: de tous ceux dont je parlerai ici, c'est celui qui illustre le mieux la conjonction entre une époque et un destin. C'est parce qu'au dernier tiers du xviI ${ }^{\mathrm{e}}$ siècle, le Québec autorisait ce type de parcours, en appelait presque l'avènement, que, dans l'anomie de ces décennies, de tels individus pouvaient s'y "faire un nom" et le perdre aussi bien. Parti de rien (Laterrière émarge tout au plus de nobliaux albigeois), l'immigrant de vingt ans devient commis, inspecteur, puis directeur des forges du

1 Jacques G. Ruelland, Pierre de Sales Laterrière. 1747-1815. Médecin et libre penseur, Longueuil, Société historique du Marigot, $\mathrm{n}^{\circ} 24,1990,88 \mathrm{p}$. 
Saint-Maurice, prisonnier politique, médecin diplômé de Harvard, marchand apothicaire, notable, juge de paix et seigneur des Éboulements ${ }^{2}$. C'est là qu'il rédige son autobiographie avant de mourir en 1815. Puis, c'est l'oubli jusqu'à la publication posthume de ses mémoires en 1873. Puis, à nouveau, l'oubli jusqu'à nos jours ${ }^{3}$. Pour un mémorialiste, quel destin! Il fut en effet le premier auteur de mémoires au Québec et le témoin privilégié d'une époque aujourd'hui sans nom ${ }^{4}$.

De quoi s'agit-il? C'est le début du régime anglais au Canada, ou plutôt dans ce que les conquérants nomment pour la première fois la "Province of Quebec" (elle deviendra en 1791 le "BasCanada"). Pour bien cerner les "agents" et leurs "pratiques", pour surtout définir ce qu'on entend par "normal " et "anormal", "typique" et "atypique", il faut d'abord préciser dans quel contexte sociohistorique, politique et culturel on navigue. Comment caractériser la société nouvelle qui se met en place au lendemain du régime français? Après le Traité de Paris (1763), les Canadiens sont confrontés pour la première fois de leur existence à une altérité généralisée. J'ai exposé ailleurs le type de monolithisme caractérisant les habitants de ce vaste territoire sous la domination française ${ }^{5}$ : émigration doublement réduite (en nombre, par rapport aux colonies anglaises et du point de vue de l'origine, principalement métropolitaine); uniformité de la langue (le Français se standardise ici plus vite qu'en France) et de la religion (catholique), contacts restreints avec les peuples autochtones (contrairement à la légende, le coureur des bois m'apparait une

2 Sur Pierre de Sales Laterrière (né en 1743 et non en 1747), voir Bernard Andrès, "La réception de l" étrange" au Québec. Pierre de Sales Laterrière (1743-1815)", dans Critique et littérature québécoise, Annette Hayward et Agnès Whitfield (dir.), Montréal, Triptyque, 1992, p. 199-216 et René Beaudoin, "Pierre de Sales Laterrière, médecin, mémorialiste et prototype de laventurier des lettres", dans Bernard Andrès (dir.), Principes du littéraire au Québec (1766-1815), Cahiers de l'ALAQ, Université du Québec à Montréal, $\mathrm{n}^{\circ}$ 2, août 1993, p. 43-56.

3 Voir la réédition en fac-simile des Mémoires de Pierre de Sales Laterrière et de ses traverses lédition intime, Québec, Imprimerie de l'Événement, 18731, Montréal, Léméac, coll. "Trésors du Patrimoine québécois ", 1980, 271 p.

4 On se déchire encore pour l'appeler la "Conquête" ou la "Cession"; voir le récent film de Jacques Godbout Le sort de l'Amérique, ONF, 1996.

5 Bernard Andrès, "Québec: paradigme littéraire et éclosion culturelle à la fin clu XvıII ${ }^{\mathrm{e}}$ siècle", Tangence, Université du Québec à Rimouski, no 51 , mai 1996, p. 67-80. 
figure atypique). Tout au plus, les dernières générations de Canadiens avant la Cession se découvrent-ils une différence par rapport aux Français de la colonie (les administrateurs et religieux de passage). Mais, comme la France n'a pas autorisé l'imprimerie, ce sentiment identitaire n'a pu se développer, s'élaborer en "opinion" et s'exprimer dans un "espace public". Quand bien même on en trouverait trace dans les écrits privés, ce sentiment identitaire ne verrait le jour qu'en regard d'un "Autre" (le Français) somme toute assez semblable au "Même" (le Canadien blanc, francophone et catholique). Avec la Conquête (ou la Cession), c'est un tout autre "Autre", l'Anglais, qui entre en scène. Ce "survenant "active et complexifie le processus de différenciation identitaire canadien. Mais cet Anglais n'est pas seulement britannique et anti-catholique. Il y a certes des marchands anglais, aventuriers sans scrupules, qui comptent bien mettre au pas les Canadiens. Mais ils n'ont pas l'oreille du gouvernement. Le "French Party" domine alors chez les nouveaux maîtres de la province. Ceux-ci sont d'ailleurs appuyés par des régiments venus d'autres états européens. Ainsi, aux yeux des Canadiens, l' Anglais" est aussi bien mercenaire suisse ou allemand et - bizarre - , il parle aussi français (car Londres favorise une administration bilingue dans cette colonie majoritairement francophone). Les "nouveaux sujets " de la Couronne sont donc confrontés à une altérité généralisée mais aussi clivée: des officiers britanniques rompus à la culture française jouent Molière en garnison, des Allemands comme le général Riedesel et sa femme la baronne salonnent en français chez les notables canadiens. Enfin, des huguenots français jadis exclus de la colonie occupent maintenant le haut du pavé. C'est dire, pour faire court, que l'anomalie règne en norme.

On le voit, tout bascule, tout change et pourtant, que de choses restent en place ${ }^{6}$ ! Car, face à la révolte des colonies américaines, Londres recule, manœuvre et compose avec les Canadiens. Pour faire carrière dans la colonie, les Canadiens doivent en principe faire le serment du Test. Mais cet acte d'allégeance religieuse et politique est loin d'être aussi contraignant qu'en Acadie, quelques années plus tôt. Et puis, bien vite, les Canadiens jouissent du libre exercice de la religion catholique et les curés

6 Sur l'effet de la cession sur l'imaginaire et les institutions canadiennes, voir le récent article de Marcel Trudel, "1760 a eu aussi ses avantages", dans Les écrits, no 89,1997, p. 21-45. 
peuvent recevoir la dîme. Le serment qui écartait les papistes des charges publiques devient inopérant. Juridiquement, les lois civiles françaises ont toujours cours; quant aux lois criminelles, le droit anglais qui s'applique à présent s'avère plus libéral que celui de l'ancienne mère patrie. Sans compter ces nouveaux lieux de fraternisation que sont les loges maçonniques. Après les loges militaires, sont venues les civiles où des marchands anglais et français peuvent se retrouver, discuter de nouvelles valeurs sociétales et philosophiques. Mais aussi négocier, tout bonnement, par-delà les clivages nationaux et religieux. Ces repaires de gens d'affaires sont autant de nouveaux repères pour les Canadiens dont la "Référence" évolue (pour reprendre l'expression de Fernand Dumont ${ }^{7}$ ). Phénomène essentiellement urbain limité à la petite bourgeoisie commerçante et professionnelle? Certes. Mais en milieu rural, la présence militaire anglaise (et allemande) est aussi généralisée. Surtout, la révolte des colonies américaines engendre une guerre de propagande qui n'épargne surtout pas les "habitants". Gustave Lanctôt et bien d'autres chercheurs ont montré la diffusion des idées révolutionnaires dans les chaumières $^{8}$ (truffés de références à Montesquieu, Locke et Voltaire, la propagande du Congrès, les appels en français, lus à haute voix, conduiront à des soulèvements sporadiques au moment de l'invasion américaine de 1775-1776). Rebelles, les Canadiens? Juste sympathiques à la cause bostonnaise? Ou globalement neutres et plutôt cyniques à l'endroit de cette guerre entre Anglais? Peu importe, de notre point de vue. Ce qui compte, c'est que la "norme" n'a jamais été aussi versatile qu'à cette époque bénie où rien, aucun appareil d'état ou religieux n'est suffisamment stable pour édicter efficacement une conduite à suivre. D'appareil, les Canadiens ne connaissent alors que l'administration coloniale anglaise qui, comme on l'a vu, mêle inextricablement les cartes juridico-ethnico-politiques, surtout durant la guerre d'indépendance américaine. Le clergé catholique, lui, n'a jamais été aussi faible numériquement et fragile comme corps constitué 9 . Cour-

7 Fernand Dumont, Genèse de la sociêté québécoise, Montréal, Boréal, 1993, p. 16-17.

8 Gustave Lanctôt, Le Canada et la Révolution américaine, Montréal, Beauchemin, 1965, 330 p. Voir aussi Michel Grenon (dir.), L'image de la Révolution française au Québec. 1789-1989, Montréal, HMH-Hurtubise, 1989, 269 p.

9 Abbé Honoré-Auguste Gosselin, L'Église du Canada après la Conquête: Première partic: 1760-1775, Québec, Laflamme, 1916, p. 1-97. 
roie de transmission du pouvoir britannique, il peine à faire respecter l'ordre parmi ses ouailles, comme parmi ses ministres. On est loin de l'empire qu'il aura sur la société canadienne au siècle suivant. Voilà donc très schématiquement le tableau socio-politique de la colonie.

Qu'en est-il du portrait "littéraire "? Une "norme" y prime-t-elle, qu'il s'agisse de genres constitués ou de traditions nationales? On sait qu'à l'époque, la notion de "nation" ne recouvre pas ce qu'elle désignera au siècle suivant. Encore moins dans les Belles Lettres où, si la France impose alors une forme d'hégémonie culturelle et linguistique, elle n'a nullement l'apanage des "Lumières". Celles-ci, comme l'a bien montré Marcel Trudel, passent aussi par l'Angleterre ${ }^{10} . \mathrm{Si}$ "norme" il y a, elle relève nécessairement de plusieurs traditions. Cela dit, quelles sont les conditions d'exercice du littéraire au Québec, qui en sont les "agents" et comment se "distinguent-ils" (pour reprendre un vocabulaire bourdieusien qui, on le verra, ne convient guère à notre cas de figure).

À l'époque, en effet - bonheur extrême - pas de grands auteurs, pas d'écoles littéraires, peu d'écoles (tout court): un collège de Sulpiciens à Montréal, le Séminaire de Québec et pas encore d'université. Une seule gazette (bilingue) à partir de 1764: la Gazette de Québec. C'est un journal officiel ne laissant qu'une portion congrue aux Belles Lettres (le "Poet Corner"). Il faut attendre 1778 pour que la deuxième gazette (unilingue française, elle) devienne littéraire. Mais en le devenant, la Gazette de Montréal vire aussi philosophique et voltairienne. Cela lui vaut, l'année suivante, la censure et l'emprisonnement de ses rédacteurs. Ces fous littéraires n'avaient-il pas profité des "douceurs" du gouvernement pour fonder une académie de province qui sentait le soufre ${ }^{11}$ ? Au trou, Jautard, le premier journaliste francophone qui signait (entre autres) "Le Spectateur tranquille" Au trou, Fleury Mesplet, le premier imprimeur francophone qui signait (notamment) "L'Impatient"! Trois ans de cachot pour méditer sur "la liberté de blâmer". Que ne s'étaient-ils pas tenus à

10 Marcel Trudel, "Le vent souffle aussi de l'Angleterre", dans Michel Grenon ot al., L'image de la Révolution française au Québec. 1789-1989, Montréal, HMH, 1989, p. 25-41.

11 Il est question de cette académie dans la Gazette littéraire de Montréal (Lettre de L.S.P.R.S.T. à l'imprimeur, 21 octobre 1778); cf. Jean-Paul De Lagrave, Fleury Mesplet (1734-1794): diffuseur des lumières au Québec, Patenaude éditeur, 1985, p. 128. 
la sage politique éditoriale de la Gazette de Québec: ne rien dire contre la religion, l'état ni le gouvernement? Tout comme le fameux barbier, les journalistes n'avaient-ils pas affirmé, en mai 1778, que la Gazette de Montréal écarterait "tout ce qui pourrait porter le moindre ombrage au gouvernement et à la religion"? Et qu'on n'y ferait même "aucune mention des affaires présentes "12. Les gazetiers n'avaient qu'à s'y tenir. Leur gouverneur Frederick Haldimand, mercenaire suisse à la solde de Londres, n'était-il pas alors l'image même du despote éclairé? Ne mettait-il pas en place, la même année, la première bibliothèque publique avec l'armada des écrits voltairiens et l'Encyclopédie de Diderot? Paraphrasons Figaro: si, dans leurs écrits, ces gazetiers n'avaient parlé "ni de l'autorité, ni du culte, ni de la politique, ni de la morale, ni des gens en place, ni des corps en crédit, ni de l'opéra, ni des autres spectacles, ni de personne qui tienne à quelque chose", ils eussent pu "tout imprimer librement [...] sous l'inspection de deux ou trois censeurs", bien sûr. Mais qui étaient ces censeurs au Québec? Il y avait d'abord Étienne Montgolfier ${ }^{13}$, supérieur des Sulpiciens qui administraient le Collège de Montréal et dont les étudiants se délectaient à la lecture de la Gazette, y collaborant à l'occasion. Il y avait aussi le père Bernard Well, jésuite et poète qui, tout en inspectant le papier périodique, ne détestait pas d'y envoyer des textes sous le pseudonyme de "L'Anonyme". Bizarre, ce jésuite: un de ses poèmes, "La chanson du jeu d'échec", peut être lu comme une charge contre le gouverneur. L'imprimeur devra lui-même le censurer pour ne pas encourir les foudres de Son Excellence ${ }^{14}$. Il y avait aussi le juge Rouville, dont les journalistes dénonçaient les abus et exactions. Il fulmina contre les "déportements de ces hommes insolents" et, le 1er juin 1779 , il obtint contre eux des lettres de cachet ${ }^{15}$.

Donc, résumons: trois censeurs (dont un censeur censuré: le jésuite Well) et deux journalistes (amenés parfois à auto-censurer

12 Maurice Lemire (dir), La vie littéraire au Québec. t. 1 (1764-1805). La voix française des nouveaux sujets britanniques, Québec, Presses de l'Université Laval, 1991, p. 240.

13 Cf. Lettre d'Étienne Montgolfier à Frederick Haldimand, 2 janvier 1779, Archives publiques du Canada, MG 21, Papiers Haldimand, B. 72-1.

14 lettre de Mesplet au secrétaire de Haldimand au sujet du père Well (4 janvier 1779; cité par Jean-Paul de Lagrave, 1985, op. cit., p. 169-170). Jean-Paul De lagrave a retracé cet épisode (op. cit., p. 169-171) Ibid., p. 172-173. 
leur feuille). Une poignée de collégiens et une vingtaine de "citoyens" de Montréal, qui pétitionnent en faveur des journalistes. Parmi eux figurent quelques "académiciens". Si l'on précise que Jautard et Mesplet se répandaient en pseudonymes dans leur gazette, mimant souvent à deux des "polémiques" (comme celle entourant la mort de Voltaire) ${ }^{16}$, le petit monde engagé dans l'aventure journalistique atteint à peine une quarantaine de personnes (pour cent abonnés à la gazette) ${ }^{17}$. Pas de quoi constituer un "champ littéraire", donc, dans cet espace public en formation. L'activité scripturale n'y dote pas encore son adepte d'un "capital symbolique" et "l'auteur" reste encore à venir ${ }^{18}$. N'y a-t-il donc plus rien à dire sur ce chapitre? Non, bien sûr. Car c'est dans ce contexte atypique où tout reste à inventer - c'est même grâce à cette absence de traditions locales - que vont s'illustrer les plus étranges individus. La question est de savoir si ce que nous percevons aujourd'hui comme étrange ou original ne fondait pas alors la norme, compte tenu de ce flou artistique propre à l'époque. Qu'on en juge.

Commençons par le petit monde ecclésiastique, le plus apte par tradition à manier la plume et à dispenser les sciences et les Lettres (lesquelles, pour beaucoup, ne font qu'un à l'époque). S'ils ne siègent plus en haut de la pyramide sociale comme sous le régime français, nous savons que le nouveau système leur confère un rôle tampon entre le "peuple" et les autorités coloniales. Ils ne constituent pas à proprement parler un appareil. Leur institution a été frappée par la Conquête, les communautés sont dissoutes ou appauvries, mais ils forment un réseau encore tenace, bien que clairsemé dans la province. C'est la seule organisation francophone dotée d'une hiérarchie qui, dès le Traité de Paris, lutte pour se reconstituer (Olivier Briand va obtenir à Londres la nomination d'un évêque que même le Vatican refusait à la province) ${ }^{19}$. Par comparaison, l'autre système hiérarchique hérité de la NouvelleFrance, l'aristocratie est elle-même ruinée: maintenue artificielle-

16 Pierre Hébert et Jacques Cotnam, "La Gazette littérare (1778-1779): notre première ceuvre de fiction?", dans Voix et images: Arcbéologie du littéraire au Québec, vol. XX, n० 2 (59), hiver 1995, p. 294-312.

17 Cela dit, toutes proportions gardées, ce lectorat est comparable à celui des gazettes curopéennes. Cf. Jürgen Habermas, L'espace public. Archéologie de la publicité comme dimension constitutive de la société bourgeoise, Paris, Payot, 1978, p. 77 et 272 , note 26.

18 L' "auteur" au sens du signataire faisant autorité sur une "cuvre" instituée.

19 Honoré-Auguste Gosselin, op. cit., p. 86-97. 
ment en place par des gouverneurs complaisants, elle s'allie parfois à la bourgeoisie commerçante. On la retrouve dans certaines loges maçonniques ${ }^{20}$, mais, sauf exception, elle n'aura pas le rôle émancipateur d'une certaine noblesse française à la même époque. L'Église, donc, va fournir les premiers écrivains. Elle s'active, pétitionne en haut lieu et intervient plus discrètement dans le réseau des communautés de l'ancienne métropole. Déjà, parmi ces clercs, apparaissent ceux que Didier Masseau appelle des "intellectuels organiques", gens de plume mettant leurs talents au service d'une officine, d'un pouvoir ou d'une organisation ${ }^{21}$. Dans le monde clérical canadien, ces individus ne défendent pas tous la même cause, peu s'en faut. Certains se dévouent corps et âme à la survie de l'Église catholique romaine. C'est le cas de Jean-Olivier Briand qui fourbit ses armes dans l'ombre du vieil évêque $M^{\mathrm{gr}}$ Dubreuil de Pontbriand et qui, à la mort de ce dernier (1760), prend les rênes du pouvoir. Briand sera nommé évêque, alors que le chapitre avait voté pour Montgolfier dont les Anglais ne voulaient pas. C'est aussi le cas de Joseph-Octave Plessis qui assiste son évêque $\mathrm{M}^{\mathrm{gr}}$ Hubert dans la crise sur le projet d'université laïque en 1789-1790. Par la suite, Plessis deviendra le pourfendeur attitré des idées révolutionnaires au Québec. C'est lui qui, avant même de devenir évêque, fourbira le mythe de la Conquête providentielle des Canadiens par les Anglais ${ }^{22}$.

Face à ces bons serviteurs de l'Église, œuvrent et manouvrent aussi des clercs tout aussi doués, mais en rupture de ban, eux. Je passe le cas des prêtres sympathiques aux rebelles américains en 1775-1776, ou simplement réfractaires au régime anglais ${ }^{23}$. Peu ou pas d'écrits chez eux. Ils n'ont laissé d'autre

20) Cf. Roger le Moine, "Francs-maçons du régime français et de la "Province of Quebec" ", dans Bernard Andrès, Principes du littêraire au Québec (17661815), Cahiers de l'ALAQ, no 2, août 1993, p. 17-33.

21 Didier Masscau, L'invention de l'intellectuel dans l'Europe du xvir' siècle, Paris, Presses universitaires de France, 1994, p. 34-38.

22 Joseph-Octave Plessis, Discours à loccasion de la victoire rempontée par les forces navales de Sa Majestée britannique dans la Méditerrannée le 1 et 2 août 1798, sur la flotte française. Ec., Québec, J. Neilson, 1799. On trouvera la plupart des textes d'époque mentionnés par cet article dans notre anthologie La conquête des Lettres au Québec (1764-1815): Florilège (en collaboration avec Pascal Riendeau), Montréal, UQAM, Département d'études littéraires, Cahiers de l'ALAQ, ${ }^{\circ} 1$, mars 1993, $446 \mathrm{p}$.

23 Dans sa notice sur Pierre Huet de la Valinière, Vincent lemieux explique ainsi le comportement de ces clercs: "L'extinction progressive des 
62

trace que l'exemple d'un sacerdoce engagé dont témoignent les objurgations et condamnations de leurs supérieurs. Le plus remarquable d'entre eux est sans conteste Pierre Huet de la Valinière ${ }^{21}$. C'est le cas pathétique, voire pathologique, de l'individu incapable de composer avec le pouvoir, quel qu'il soit. Surnommé le "Bostonnais", ou "le Juif errant du Canada", il est accusé de sympathies pour les Américains: "conduite peu modérée", "esprit farouche", "rebelle de cœur, [...] ardent, factieux et turbulent" sont les doux épithètes dont on le gratifie ${ }^{25}$. Mais le plus touchant, c'est qu'il passe ensuite toute sa vie à se justifier auprès des autorités religieuses et civiles, tant anglaises que françaises et américaines. Lettres, mémoires, poèmes autobiographiques jalonnent l'impossible carrière de ce Sulpicien d'origine nantaise. Semoncé par son supérieur Montgolfier pour avoir entaché l'honneur des Sulpiciens, il est menacé d'interdit et d'exil en France. Rien n'y fait. Bien que l'évêque lui conseille de ne pas tenter de "se justifier ni [de] faire des esclandres!", il laisse circuler la pétition de cinq cent soixante-quinze paroissiens qui n'hésitent pas à l'appuyer. Bientôt, en 1779, le gouverneur Haldimand décrète sa déportation en Angleterre. Mais le bouillant curé passe aussitôt en France. En 1781, il soumet au comte de Vergennes, ministre des Affaires étrangères, un projet d'insurrection du Québec. Selon lui la province peut être reconquise par la France. Mais, comme le dit son biographe, "La signature du traité de Versailles en 1783 anéantit tous les espoirs de ce patriote invétéré. " ${ }^{26}$ Interdit de séjour chez les Sulpiciens de Paris, il se réfugie chez ceux de sa

communautés religieuses avait fait l'objet d'une instruction royale difficile à accepter de la part des jésuites, des récollets et même des sulpiciens. Il n'est donc pas trop surprenant de trouver parmi eux des prêtres réfractaires au nouveau régime politique: les jésuites Joseph Huguet, Bernard Well et Pierre-René Floquet, le récollet Claude Carpentier et l'ex-récollet Eustache Chartier de Lotbinière. La Valinière aurait penché du même côté l...l", Vincent Jemicux, "Pierre-Huet de La Valinière", Dictionnaire biograpbique du Canada, vol. V, Sainte-Foy, Presses de l'université Laval, p. 475-477.

24 Pierre Huct de La Valinière est l'auteur de Dialogue curieux et intênessant entre Mr. Bun Désir et le Dr. Breviloq en français et en anglais, ou l'on peut aisément trouver les armes pour défendre la religion contre toutes les faussetés inventées contre elle (New York, 1790), qui est aussi paru en anglais, et cle Vraie Histoire, ou Simple Précis des infortunes, pour ne pas dire, des persécutions qu'a souffert \& souffre encore le révérend Pierre Huet de La Valinière I...I (Albany, N.Y., 1792).

25 Vincent l.emieux, op. cit., p. 476

26 Ibid. 
Nantes natale. L'esprit d'aventure le reprend alors et à cinquantetrois ans, il regagne l'Amérique. Le Québec lui est toujours fermé, aussi exerce-t-il son ministère à New York, puis dans les Illinois où, pendant trois ans, il se chicane avec ses ouailles et les curés voisins. De retour à New York, il déclenche encore l'ire des paroissiens qui mettent le feu à son église et à son presbytère. En 1792, il obtient enfin le pardon des Britanniques et finit sa vie misérablement, mendiant sa pitance auprès de ses anciens paroissiens (car l'évêché l'a privé de cure). Il se battra jusqu'à la mort pour faire reconnaître publiquement son innocence. Parmi les écrits de ce flamboyant christique et détraqué, signalons la Vraie Histoire, ou Simple Précis des infortunes, pour ne pas dire, des persécutions qu'a souffert $\xi$ souffre encore le révérend Pierre Huet de La Valinière [...] (Albany, N.Y., 1792), et surtout: le Dialogue curieux et intéressant entre Mr. Bon Désir et le Dr. Breviloq en français et en anglais, ou l'on peut aisément trouver les armes pour défendre la religion contre toutes les faussetés inventées contre elle (New York, 1790).

Fort intéressants au plan littéraire sont ces récalcitrants, ces transfuges qui, à la faveur du "flou sociétal" créé par le glissement d'allégeance, ont osé critiquer leur hiérarchie. Car la plupart n'ont nullement le sentiment de trahir leur communauté, qu'il s'agisse de la communauté religieuse ou de la communauté "nationale". Où est la "nation", quelle est la "patrie" de ces clercs nés en France ou au Canada, devenus "nouveaux sujets" britanniques par les hasards de la Providence et charriés par le destin d'un espace culturel à l'autre?

Voici, si j'ose dire, l'exemple type d'un atypique Canadien de naissance: Charles-François Bailly de Messein 27 . Sa famille fortunée lui permet d'aller étudier à Paris au collège Louis-Le-Grand, vers la fin du régime français. Il retourne au Québec sous le régime anglais et devient missionnaire en Acadie. Ensuite, sa trajectoire cléricale est toute tracée: professeur de rhétorique au petit séminaire de Québec, il en devient directeur. Il est remarqué par le gouverneur Carleton et le voilà précepteur de ses enfants. La tradition, dit Galarneau, le montre "vêtu d'une soutane de soie", allant et venant "du séminaire au château Saint-Louis dans le carrosse" de Son Excellence. Opposé à l'invasion américaine

27 Cf. Claude Galarneau, "François Bailly de Messein", Dictionnatre biographique du Canada, vol. IV, Sainte-Foy, Presses de l'université Laval, p. 45-48. 
64

de 1775, il est blessé par les Bostonnais qui le font prisonnier. Il regagne ensuite Québec où il ne reste pas longtemps simple curé. Cette fois, c'est à Londres que le gouverneur Guy Carleton l'emmène prendre soin de ses enfants (1778-1782). Quatre ans d'une existence princière, après lesquels il doit reprendre sa petite cure de Pointe-aux-Trembles. Mais le retour du gouverneur, en 1786 , le propulse à l'avant-scène religieuse et politique. Poussé par Lord Dorchester, il devient co-adjuteur de Mgr Hubert (au désespoir de ce dernier qui ne prise guère les grands airs de Charles-François Bailly de Messein) ${ }^{28}$. C'est alors que son parcours dévie du tout au tout. Au lieu d'attendre patiemment que la mitre lui êchoie, il se permet de contredire son évêque. Une grande enquête est lancée en 1787, "sur l'objet d'augmenter les moiens d'education" dans la province. Peu après, les Anglais agitent l'idée d'une université neutre au plan religieux, où professeraient des "hommes sans préjugés"! Consulté sur le sujet, $\mathrm{Mgr}$ Hubert s'oppose farouchement à l'entreprise qui échapperait bien sûr à sa gouverne. Les Canadiens ne sont pas mûrs pour une telle institution, argue-t-il ; nos collèges et séminaires suppléent amplement aux besoins des habitants. Que non! se permet de tonner le co-adjuteur dans une lettre qui sera bientôt publiée ${ }^{29}$. Avec un brio décapant, une ironie cinglante, Bailly de Messein réduit à néant l'argumentation de son supérieur. Le professeur de rhétorique trouve dans son pamphlet des accents voltairiens pour fustiger la barbarie et appeler de ses vœux les bienfaits de la science. Se moquant de l'évêque dont il fait un "proto-défenseur de l'ignorance au dix-huitième siècle", il en retourne une à une chaque phrase: "Le tems n'est pas venu d'établir une Université : C'est à dire faire luir le Soleil de la Science sur les jeunes Canadiens; leurs yeux sont trop faibles; il faut même opposer et élever des nuages pour en obscurcir jusqu'aux moindres rayons. "30

Ainsi, quelques mois après l'annonce de la Révolution française, paraît ici un texte qui soulèvera dans les gazettes le premier débat de fond sur l'instruction publique. Si l'on ajoute que cet appel aux "citoyens" du Canada mentionne le nom d'un

28 Nommé évêque in partibus infidelium de Capsa en septembre 1788, il est sacré évêque le 12 juillet 1789 par $\mathrm{M}^{\mathrm{gr}}$ Hubert; cf. Claude Galarneau, ibid.

29) Francois Bailly de Messein, Copie de la lettre de l'évếque de Capsa coadjuteur de Québec, É c. au président du Comité sur l'éducation, Québec, Samuel Neilson, 1790 . 
franc-maçon notoire, Simon Sanguinet, comme généreux donateur de ladite université laïque, on mesure l'émoi que cause alors l'intempestif Bailly de Messein. On imagine aussi le peu de succès de sa sortie et le triste sort qui lui fut fait par la suite. S'il ne connut pas la misère de Huet de la Valinière, il souffrit quand même toutes les misères qu'une Église offensée ne manqua pas de lui infliger. Inutile de préciser que, de la mitre, il ne vit pas la couleur.

À côté de ce destin tragique, les turbulences d'autres petits clercs font sourire. C'est le cas de ceux qui, jouant encore sur les changements de "normes" et de "référence ", ont carrément défroqué ou apostasié. Ainsi du Frère Berthiaume, Récollet, apostat et coureur des bois (ou de jupons indiens). Fuyant le couvent de Montréal "en compagnie de deux ou trois sauvages", il se réfugie chez eux où "il a la hardiesse de confesser et de dire [...] la messe en blanc". Poursuivi par les Franciscains, il leur échappe jusqu'en Acadie où, dit la chronique, "il continua [son] œuvre diabolique "31. Ainsi, également, du père Veyssière, Récollet. Prenant pour acquis la liberté nouvelle accordée aux prêtres par les Anglais, Veyssière apostasie publiquement en 1766 . Selon la formule consacrée, il "embrasse le saint état du mariage"sans redouter aucune peine émanée du siège de Rome. Et il devient incontinent ministre du culte protestant (1767) ${ }^{32}$. Mais le pire (que l'historiographie cléricale voile pudiquement), c'est qu'en 1771 , il est devenu franc-maçon et qu'on le propose Maître d'une première loge civile à Trois-Rivières ${ }^{33}$.

Le plus beau des cas limites est celui de Pierre Roubaud, jésuite défroqué. J'ai évoqué ailleurs ses joyeux méfaits et Caroline Masse lui a consacré un récent mémoire ${ }^{34}$. Qu'il suffise

31 Honoré-Auguste Gosselin, L'Église du Canada après la Conquête: Deuxième partie: 1775-1789, Québec, Laflamme, 1917, p. 161.

32 Sur cet épisode de la vie de Leger Jean- Baptiste Noël Veyssiere, cf. HonoréAuguste Gosselin, op. cit., p. 251.

33 A. J. B. Milborne, Freemasonry in the Province of Quebec: 1759-1959, Knowlton, Québec, [s.n.], 1960, p. 31.

34 Bernard Andrès, "Du faux épistolaire: Pierre-Joseph-Antoine Roubaud et les Lettres de Monsieur le Marquis de Montcalm [...] écrites dans les années 1757 , 1758, 1758", dans Georges Bérubé et Marie-France Silver éditeurs, La lettre au $x$ virf siecle et ses avatars, Toronto, Éditions du GREF, coll. "Dont actes", $\mathrm{n}^{\circ} 14,1996$, p. $231-248$ et Caroline Masse, "Pierre Roubaud, faussaire polygraphe au Siècle des lumières", dans Voix et images, $\mathrm{n}^{\circ} 59$, hiver 1995, 
66

de rappeler brièvement que le changement d'allégeance a littéralement catapulté cet obscur missionnaire au coeur de l'actualité politique. L'arrivée du conquérant convient parfaitement à cet esprit retors qui vole offrir ses services aux nouvelles puissances. Ses excès de zèle gênent même à ce point les Anglais qu'ils l'engagent plutôt à quitter la province: il sera plus utile à Londres. Trompant alors l'indulgence des jésuites de Québec, il obtient d'eux qu'ils paient son passage (ils lui accordent même un viatique pour aller se retirer, pensent-ils, dans un couvent européen). Mais c'est à Londres qu'il s'installe (1764). Bien décidé à monnayer ses connaissances de la colonie auprès du comte de Halifax, secrétaire d'État pour les départements du sud, il multiplie les mémoires dans le sens voulu. Comment assimiler les Canadiens? Il suffit de les éloigner de la religion catholique, d'empêcher la nomination d'un évêque, de réduire le clergé, de confisquer les biens des jésuites, etc. (l'homme espère bien tirer une ristourne de cette dernière opération). George III lui accorde une pension. Mais au gré des changements de ministère, l'exjésuite doit continuellement reprendre ses démarches, s'assurer des appuis, écrire sans fin des mémoires en faisant miroiter à ses protecteurs le parti qu'il pourront tirer de sa collaboration. Je passe sur sa vie dépravée, son mariage, ses emplois de comédien et toute la panoplie des expédients dont il use dans le monde interlope de la bohême londonienne. Son fait d'arme le plus connu est un faux. Il forge de toutes pièces des lettres que le marquis de Montcalm aurait écrites à la veille de la déconfiture française, entre 1757 et 1759 . Le général aurait prévu très tôt la défaite, la stratégie de la bataille fatale, sa mort sur les plaines d'Abraham et surtout, la révolte des colonies américaines (!). Comme par hasard, ces lettres sont publiées à Londres en 1777 , en pleine guerre d'indépendance américaine. Si des doutes apparaissent bientôt sur l'authenticité des épîtres, l'opération réussit quand même à semer l'émoi, de Londres à Paris. Roubaud passera le reste de sa vie à se défendre de les avoir écrites et à se vanter de les avoir forgées, selon qu'il aura affaire aux Français ou aux Anglais. Car il monnaie toujours ses services au plus offrant, agent double et même triple, puisqu'il manigança

p. 314-328, ainsi que "Le faux et la contrefaçon: Pierre Roubaud, polygraphe et faussaire au siècle des lumières (1723-c. 1789)", mémoire de maîtrise (B. Andrès, directeur), Département d'études littéraires, Université du Québec à Montréal, 1996, 176 p. 
quelque temps pour l'Espagne. Et quand des Canadiens se rendront à Londres pétitionner contre les exactions d'un gouverneur, le zélé transfuge les aidera dans leurs démarches au parlement (tout en les dénonçant dans l'ombre auprès des ministres concernés). Parmi ces victimes, Pierre du Calvet qui, après trois ans de prison sous le gouverneur Haldimand, était venu à Londres réclamer un procès contre le "tyran": il y publia un pamphlet politique dans les deux langues, Appel à la justice de l'État, ou The case of Peter du Calvet (1784). On dit que Roubaud participa à la rédaction ${ }^{35}$.

Nul doute que ce Roubaud compte parmi les "intellectuels organiques " dont Didier Masseau parle en évoquant la carrière de Théveneau de Morande à Londres, parmi les espions de la police française ${ }^{36}$. Dans un tel paradigme, Roubaud n'est nullement atypique. L'est-il même par rapport au "modèle jésuite" (s'il en existe un)? En effet, bien que la Compagnie ait fini par l'exclure de ses rangs, scotomisant notamment son nom dans les fameuses Lettres édifiantes et curieuses ${ }^{37}$, on sait aussi comment les bons pères s'accommodaient parfois d'impératifs antinomiques. Ou pour le dire comme un de leurs anciens élèves: "Pour moi, je ne vois rien de si divin que Los Padres, qui font ici [au Paraguay] la guerre au roi d'Espagne et au roi du Portugal, et qui en Europe confessent ces rois; qui tuent ici des Espagnols, et qui à Madrid les envoient au ciel; cela me ravit [...]. " "38 Mais laissons-là Voltaire qui leur reconnaissait aussi candidement des mérites et, convenons simplement que Roubaud n'est pas très atypique. Il en est autrement des derniers personnages que j'aborde ici: des laïcs, ceux-là.

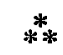

35 Cf. Auguste Vachon, "Pierre-Joseph-Antoine Roubaud ", Dictionnaire biographique du Canada, tome IV, Sainte-Foy, Presses de l'université Laval, p. 74345.

36 Didier Masseau, op. cit., p. 34-38.

37 Abbé Patouillet (êd.), Lettres édifiantes et curieuses écrites des missions étrangères par quelques missionnaires de la $C$. de J., Paris, Chez Charles Pierre Berton, recueil XXXIII, 1776, p. 210- 297

38 Voltaire, Candide ou L'opimiste, Paris, Classiques Garnier, édition H. Bénac, 1759, chapitre 14 , p. 167. 
68

Parallèlement aux clercs impliqués dans la chose publique, des laïcs interviennent aussi dans les débats de société au Canada. La plupart sont des notables inféodés au pouvoir: des loyalistes comme le fonctionnaire François Baby, le marchand Alexandre Dumas ou les notaires Jean-Baptiste Badeaux et Simon Sanguinet ${ }^{39}$. Pour trouver des individus en rupture de ban, il faut chercher chez les immigrés français des années 1760-1770, comme les gazetiers Jautard et Mesplet, le médecin Pierre de Sales Laterrière, le pamphlétaire Pierre du Calvet, ou le poète Joseph Quesnel. L'Américain d'origine française Jean/John de Crèvecour est un cas bien à part qui mériterait à lui seul un volume ${ }^{40}$. On peut aussi trouver des destins marginaux chez des Canadiens émigrants comme Jacques Grasset de Saint-Sauveur ou chez le jeune révolutionnaire Henry-Antoine Mézière ${ }^{41}$. Canadiens ou français, ces personnages partagent l'expérience de la migration et d'un certain cosmopolitisme. Il n'est pas jusqu'au gouverneur Frederick Haldimand qui, par son statut de mercenaire suisse inféodé à Londres, ne puisse figurer dans notre cabinet des curiosités. À cheval sur deux nations et (au moins) autant de systèmes de valeurs, tous ces gens transgressent une norme dans cette province elle-même marquée par une forte versatilité culturelle, on l'a vu. Je ne reviendrai pas sur les journalistes Jautard et Mesplet, ni sur du Calvet dont je traite ailleurs plus longuement ${ }^{42}$. Le cas de Joseph Quesnel appelle quelques commentaires. Quand il arrive en 1779 , il a déjà voyagé aux Indes, à Madagascar, aux Antilles et au Brésil. Royaliste dans l'âme, ce malouin sera toujours un bon sujet de la couronne. C'est dans le domaine culturel que détonne quelque peu

39 On trouvera la plupart de ces auteurs dans notre anthologie La Conquête des Lettres au Québec (1764-1815): Florilège, op. cit. Pour ce qui est de Simon Sanguinet, seuls son rattachement à la franc-maçonnerie et son rôle posthume dans le débat sur l'université publique font de ce dernier un personnage allodoxique (aux yeux du haut-clergé catholique).

40 Michel Guillaume Crèvecour, Lettres d'un cultivateur américain écrites à W.S. Ecuyer: depuis l'année 1770, jusqu'à 1781, Paris, Cuchet, 1784. Cf. Warren Barton Blake, Introduction à la réédition des Letters, New York, Dutton, 1957.

41 Sur Jacques Grasset de Saint-Sauveur, voir Pierre-Georges Roy, Les petites choses de notre histoire, Lévis, 1922, p. 265-268; nous reviendrons sur HenryAntoine Mézière.

42 Bernard Andrès, "La passion du combat dans les lettres de Pierre du Calvet (1779-1784)", colloque "Érudition et passion dans les écritures intimes", $65^{\mathrm{e}}$ Congrès de l'ACFAS, Université du Québec à Trois-Rivières, 12 mai 1997 (à paraître dans les actes). 
ce riche marchand de fourrures qui se retire à quarante-six ans pour se consacrer à la poésie et tenir salon à... Boucherville. Entre-temps, il a monté la première troupe de théâtre francophone, le "Théâtre de société", avec des amis francs-maçons ${ }^{43}$. Le philanthrope musicien tenait aussi l'orgue à l'Église de Montréal, au grand dam du curé qui trouvait trop allègre sa partition:

\section{[...] On traita de folâtre}

ma musique, dit-on, faite pour le Thêâtre:

L'un se plaint qu'à l'Église il a presque dancé

L'autre dit que l'auteur devrait être chassé;

Chacun tire sur moi et me pousse des bottes,

Le sexe s'en mêla et surtout les dévôtes. ${ }^{44}$

Dans le débat qui fit rage sur les méfaits du théâtre, alors que l'Église condamnait (encore) les spectacles, Quesnel n'hésita pas à défendre son art dans la Gazette de Montréal (sous le pseudonyme d'“Un acteur" $)^{45}$.

À la même époque, un jeune étudiant de Montréal, Henri Mézière, rue aussi dans les brancards. Il ira bien plus loin que Quesnel dans la dénonciation, puisqu'il deviendra, lui, l'ardent défenseur de la Révolution française. On le soupçonne d'avoir publié chez Fleury Mesplet le pamphlet La Bastille septentrionale $(1791)^{46}$. Voici comment, dans une lettre à ses parents, il raconte son séjour chez les Sulpiciens: "un collège confié à d'ignares ecclésiastiques fut le tombeau de mes jeunes ans, j'y puisai quelques mots latins et un parfait mépris pour mes professeurs " ${ }^{47}$.

Exilé volontaire aux États-unis (1793), le jeune turc propose ses services à Edmond-Charles Genêt, ministre du gouvernement

43 Bernard Anclrès, "Archéologie de la comédie et du théâtre lyrique au Québec: Joseph Quesnel (1746-1809)", Artexto, Revista do Departamento de Letras et Artes, Fondaçào Universidade do Rio Grande (FURG, Brésil), n 8, 1997, p. 11-26.

44 Jeanne d'Arc Lortie, Pierre Savard et Paul Wyczinsky (dir.), Les textes poétiques du Canada français, 1606-1806, t. II, Fides, 1989, p. 445.

45 Gazette de Montréal, 7 janvier 1790.

46 Cf. Isabelle Beaulé, "Henti-Antoine Mézière: d'épistolier à pamphlétaire?", mémoire de maîtrise (Bernard Andrès, directeur), Département d'études littéraires, Université du Québec à Montréal, 1996. Je tire de ce travail les extraits de correspondances qui suivent.

47 Henri-Antoine Mézière, "Lettre de Henri Mézière à ses parents de Cumberland Head, le 28 août 1793", Fonds Verreau, 17, no 32, Archives du Séminaire de Québec, 5 p. 
révolutionnaire auprès du Congrès. Dans un mémoire sur les dispositions politiques des Canadiens, Mézière prétend que ses compatriotes sont prêts à se soulever contre le "despotisme et la tyrannie" anglaises. Voici un aperçu de son style épistolaire:

Tôt ou tard, écrit-il à ses parents, la liberté régnera dans le monde entier; bientôt ce servile univers, mû par ce contact électrique, sortira de son sommeil de mort et se réveillera république. Alors le fils ne fuira plus le père pour se soustraire à la tyrannie. Ce sera l'âge d'or, le siècle d'Astrée et de Rhée. Ainsi soit-il. ${ }^{48}$

Mais devant l'apathie du "peuple canadien", et le peu d'empressement de la France à envahir le Québec, il s'exile à Paris où il tombe, le malheureux, au pire moment de la Terreur. Prison, fuite en province, refuge à Bordeaux... où on le retrouve vingt ans plus tard fonctionnaire municipal. Je n'ai pas le loisir de poursuivre sur son retour au Québec. Il suffit de rappeler que, bien qu'aux antipodes idéologiquement, Mézière comme Quesnel poussent le goût du risque jusqu'à rompre avec leur milieu. Chez eux comme chez du Calvet, Jautard et Mesplet, on note cet aspect suicidaire du comportement qui fait tout le sel de leur déviance.

Dans cet espace culturel encore embryonnaire qui ne dote pas encore l'"intellectuel " d'un "capital symbolique", ces francstireurs ont tout à perdre: au Québec alors, le temps n'est pas encore venu de prendre des postures et de tirer prestige d'un beau geste. Il n'y a pas encore de public pour consacrer le héros; à peine quelques tribunes. Et des prisons, oui. Ou l'exil. Ou la mort en mer (comme pour du Calvet qui périt mystérieusement au cours d'une traversée vers Londres où il partait une fois encore à l'assaut des moulins). La plupart du temps, c'est l'oubli qui attend ces "O.L.N.I." ${ }^{49}$. Un long purgatoire est le lot de ces êtres hors-normes qui font le seul délice d'universitaires décadents en mal d'allodoxies passées ${ }^{50}$.

Mais, demandons-nous, M.A.D.O.N.N.A. : et s'il y avait maldonne? Et si ces cas ne nous intéressaient que pour le crédit dont nous jouirions à leur accorder quelque crédit? Et si nous n'étions là que pour consacrer à travers eux notre propre allodoxie? Le

\footnotetext{
48 Ibid.

49) Objets latéraux non identifiés.

50 U.D.M.A.P.
} 
programme de ce colloque parlait de "l'inscription presque perverse de ces marginalités" dans les textes les plus consacrés. Quoi de plus consacrant que ce colloque pour y inscrire notre propre perversion, chers collègues? À moins que je ne parle qu'à mon bonnet en évoquant mes originaux et détraqués, ces êtres que l'abbé Camille Roy stigmatisait de la sorte: "[...] ces hommes à réputation louche, [...] ces demi-lettrés et [...] ces épaves de la morale que le flot de la mer avait déjà jetés sur nos rivage. " ${ }^{51}$ Qu'eût-il pensé des Canadiens qui sévissaient alors en France, tels Henry-Antoine Mézière ou Jacques Grasset de Saint-Sauveur? Si le premier avait tâté de la Révolution, l'autre s'était essayé dans le libertinage avec des titres comme Les amours du fameux comte de Bonneval, pacha à deux queues ou Les amours d'Alexandre et de Sultane Amazille ${ }^{52}$. Ainsi donc, des Canadiens exilés en France se retrouvent-ils dans l'opprobre avec les "épaves" françaises échouées au Québec. Souhaitons du moins que leur renflouage aura, deux siècles plus tard, contribué à l'archéologie de l'allodoxie québécoise.

51 Camille Roy, Nos origines littéraires, Québec, L'Action sociale, 1909, p. 68.

52 Jacques Grasset de Saint-Sauveur, Les amours du fameux comte de Bonneval, pacha à deux queues, connu sous le nom d'Osman, d'après quelques mémoires particulies, Paris, 1796 , un volume in-18 et Les amours diAlexandre et de Sultane Amazille, Jaris, 1797, 2 volumes in-18. 